\title{
A Picture is Worth 1,000 Words
}

\section{The Use of Clinical Images in Electronic Medical Records}

Angela C Ai ; Francine L Maloney²; Thu-Trang Hickman¹; Allison R Wilcox ${ }^{3}$; Harley Ramelson ${ }^{1,4}$; Adam Wright ${ }^{1,4}$

${ }^{1}$ Brigham and Women's Hospital, Harvard Medical School, Boston, MA;

${ }^{2}$ Ariadne Labs at Brigham and Women's Hospital and Harvard T.H. Chan School of Public Health, Boston, MA;

${ }^{3}$ Dartmouth-Hitchcock Medical Center, Lebanon, NH; 4Partners HealthCare, Boston, MA

\section{Keywords}

Electronic health records, clinical images

\section{Summary}

Objective: To understand how clinicians utilize image uploading tools in a home grown electronic health records (EHR) system.

Methods: A content analysis of patient notes containing non-radiological images from the EHR was conducted. Images from 4,000 random notes from July 1, 2009 - June 30, 2010 were reviewed and manually coded. Codes were assigned to four properties of the image: (1) image type, (2) role of image uploader (e.g. MD, NP, PA, RN), (3) practice type (e.g. internal medicine, dermatology, ophthalmology), and (4) image subject.

Results: 3,815 images from image-containing notes stored in the EHR were reviewed and manually coded. Of those images, $32.8 \%$ were clinical and $66.2 \%$ were non-clinical. The most common types of the clinical images were photographs (38.0\%), diagrams (19.1\%), and scanned documents (14.4\%). MDs uploaded $67.9 \%$ of clinical images, followed by RNs with $10.2 \%$, and genetic counselors with $6.8 \%$. Dermatology (34.9\%), ophthalmology (16.1\%), and general surgery (10.8\%) uploaded the most clinical images. The content of clinical images referencing body parts varied, with $49.8 \%$ of those images focusing on the head and neck region, $15.3 \%$ focusing on the thorax, and $13.8 \%$ focusing on the lower extremities.

Conclusion: The diversity of image types, content, and uploaders within a home grown EHR system reflected the versatility and importance of the image uploading tool. Understanding how users utilize image uploading tools in a clinical setting highlights important considerations for designing better EHR tools and the importance of interoperability between EHR systems and other health technology.

\section{Correspondence to:}

Adam Wright, PhD

Division of General Internal Medicine

Brigham and Women's Hospital

75 Francis St.

Boston, MA 02115

Phone: (617) 525-9811, Fax: (617) 732-7072

Email: awright5@partners.org

\author{
Appl Clin Inform 2017; 8: 710-718 \\ https://doi.org/10.4338/ACI-2016-10-RA-0180 \\ received: October 20, 2016 \\ accepted: April 22, 2017 \\ published: July 12, 2017 \\ Citation: AC Ai, FL Maloney, T-T Hickman, AR Wilcox, \\ H Ramelson, A Wright. A picture is worth 1,000 words: \\ The use of clinical images in electronic medical rec- \\ ords. Appl Clin Inform 2017; 8: 710-718 \\ https://doi.org/10.4338/ACI-2016-10-RA-0180
}




\section{Background}

Electronic health record (EHR) systems have revolutionized the accessibility of diagnostic information for informed clinical decision making. While there has been a lot of focus on structured and narrative documentation in EHR, there has been less focus on non-text documentation in clinical notes. Images serve a wide range of clinical purposes, and imaging data are frequently produced and/or used by many clinicians [1]. Although most images come from the radiology department, non-radiological medical images integrated into patients' medical records can also be a critical component of documentation and information supporting clinical diagnoses [2]. Additionally, clinical pictures are useful for educational purposes [3]. In a study conducted by Lyon and Harrison, the usefulness of non-radiological images in diagnoses, especially in instances of tele-dermatology, was highlighted [4]. Since the Lyon and Harrison study, digital photographs have been increasingly important in recording visual data, particularly in pathology, dermatology, and plastic surgery [5]. A recent analysis of digital images in a surgical setting showed that ad-hoc workflows develop to include images while evaluating patients, highlighting their importance in EHRs [6]. While the utility of images is clear, the type and content of images in the EHR has not been completely explored.

Partners HealthCare System (PHS) implemented an image uploading system into the Longitudinal Medical Record (LMR), its legacy home-grown outpatient EHR. When entering notes, providers are able to insert an image into a note using an image-uploading tool, seen in - Figure 1, and can also paste images from the clipboard. In addition to uploading images, users can insert an annotated diagram from a built-in library of images. This library of images supports multiple specialties and gives the option to add specialty-specific markers to the diagram. Additionally, diagrams can be created in a different program and uploaded into the note. Graphs can also be created in a different program and then uploaded into the relevant note. While there is an option to directly upload a scanned document in as a note in the LMR, scanned documents could also be attached to the note as an image.

\section{Objective}

To better understand how the image uploading system was being utilized across PHS, we conducted a content analysis of notes containing images for patients in a quaternary academic hospital, Brigham and Women's Hospital (BWH), and developed a coding system for analyzing images in EHRs that can be used to glean valuable insight about the use of images and image systems.

\section{Methods}

We conducted a content analysis of a sample of images uploaded to the LMR for patients seen at Brigham and Women's Hospital (BWH) between July 1, 2009 and June 30, 2010. BWH is a 777-bed teaching hospital in Boston with a large collection of affiliated primary care and specialty practices. We created a database of notes containing one or more clinical images (238,450 notes). From this database, all images from a random sample of 4,000 notes was reviewed and manually coded by two research assistants. Codes were assigned to four properties of the image: (1) image type, (2) role of image uploader (e.g. MD, NP, PA, RN), (3) practice specialty (e.g. internal medicine, dermatology, ophthalmology), and (4) image subject. Examples of images and image coding are seen in $>$ Figure 2 . At the time of chart review, patients were excluded from the study if they were under the age of 18; if access to their electronic medical record was not permitted because they received care primarily at another facility in our health system; if they were a test patient; or if there were no images associated with a specified note in their record due to the notes' removal ( Figure 3). The study was reviewed and approved by the PHS Human Subjects Research Committee.

\section{Results}

We analyzed notes containing any image over a one year period from 4,000 outpatient records. Records were removed from the study if they met exclusion criteria ( $>$ Figure 3 ). A total of 3,815 images 
from image-containing notes stored in the EHR system were reviewed and manually coded ( $>$ Figure 3). When analyzing image types, we found that the images fell into two distinct groups: nonclinical images and clinically relevant images ( $\$$ Table 1 ). Non-clinical images included logos, signatures, and icons. Although this use of images was not anticipated when the new feature was added, several clinics updated their note templates to include a hospital or clinic logo, and some providers also added an image of their signature to their note templates. In fact, $66.2 \%$ of the images were nonclinical, and $91.2 \%$ of these nonclinical images were logos. We disregarded non-clinical images for our subsequent analyses. For the 1,288 clinical images, photographs (38.0\%), diagrams (19.1\%), scanned documents (14.4\%), and graphs (11.0\%) comprised the majority. Clinically-relevant images also included screenshots, tables, and forms. Notably, many of the notes contained multiple images ( Figure 3), and a large majority of those were multiple pictures of a single site.

In addition to categorizing the image type, we inspected what was being included in the clinical images. The most photographed body region was the head and neck region (297 images, 49.6\% of all photographs). Additionally, when examining the contents of the diagrams, we found that about half of them were eye diagrams $(50.6 \%)$. These eye diagrams were generally fundus diagrams, documenting the interior surface of the eye that opposes the lens. Of the 168 scanned documents, 94 were First Look documents, 30 were pathology reports, 20 were EKGs, and 6 were consent forms. First Looks are the results of noninvasive screening of the developing fetus. Of the 126 screenshots, most were screenshots of pedigrees (38 images), of optical coherence tomography (OCT) studies (28 images), or of lab results (6 images).

To further examine and characterize the clinical utilization of the image uploading system, we analyzed the types and specialties of providers who uploaded clinical images. We found that the majority of the clinical images were uploaded by people holding MDs (67.9\%). The list of providers who uploaded images also included RNs (10.2\%), genetic counselors (6.8\%), MAs (4\%), ODs (2.4\%), and NPs (2.7\%). $5.5 \%$ of analyzed images were uploaded by people with other roles. $>$ Table 2 shows the top 10 specialties of the 58 specialties at Partners that uploaded clinical images. Within our database, dermatology uploaded the most images (450 images), accounting for $34.9 \%$ of clinical images, followed by ophthalmology (16.1\%), general surgery (10.8\%), obstetrics and gynecology (5.4\%), and oncology (5.0\%). Dermatology primarily uploaded photographs (295 images), ophthalmology primarily uploaded general surgery pain scales (124), obstetrics and gynecology mainly uploaded scanned First Look documents (59 images), and oncology mainly uploaded cervix diagrams (25 images).

\section{Discussion}

The EHR is a powerful tool that can be used to examine a patient's health over the course of time. While primarily text-based, clinical documents may contain images that allow for better descriptive documentation of a person's condition, how an illness may progress over time, and may lead to improved diagnostic accuracy [4, 7]. PHS created an image uploading system in their home-grown EHR that allows providers to upload non-radiological images to supplement their patient records. To examine how clinical providers utilized images in medical records, we examined and characterized images from patient notes at one academic medical center. Our analyses showed that the majority of images (69\%) were non-clinical and, of the clinical images, the most common types were photos, diagrams and scanned documents ( Table 1 ). Physicians were the most common type of provider who uploaded clinical images; almost two-thirds of the clinical images were uploaded by physicians. Almost a third of the clinical images were dermatology related, and the next most common specialties were ophthalmology (17\%) and general surgery (13\%) ( Table 2).

We observed several trends related to the use of images in the EHR. For example, while the original intent of the imaging system was to allow users the ability to upload relevant clinical photographs to the EHR, the majority of images were actually non-clinical in nature, such as logos in the headers of notes or signatures at the bottom of referral letters. While these images may have been auto-generated by the system or have been set up as part of a note template, it is clear that these images are being used to make the electronic notes mimic physical notes. One reason for the use of logos and signatures in notes is that workflow was streamlined by their use. For example, if the patient asked for a copy of documentation, a receptionist could utilize the system and print off the note 
without needing to ask the physician to re-sign it. Another reason could be that these images were used to enhance the legitimacy and authenticity of the electronic notes. Skeuomorphism is a design concept where electronic items resemble their "real world" counterparts. The thought behind this is that familiarity aids in the transition between technologies [8]. By making the interface feel familiar, the designer encourages quicker adoption by reducing the intimidation barriers [9]. It is possible that users are unconsciously following this design principle while utilizing the EHR and the addition of images in notes both enhances the note-viewing experience and allows for user engagement.

Interestingly, screenshots were the fourth most common clinical image type. The prevalence of screenshots illustrated the issue of interoperability between clinical information systems and screenshots of pedigrees, retina studies, lab results, and optical coherence tomography (OCT) studies. The programs that were used to perform these studies or draw such diagrams are not easily integrated within the PACS systems or within the EHR, leading to workarounds to include this data in the chart. For example, users were able to work around the existing interoperability barriers to genetic medicine integration within the EHR by utilizing the image uploading system and taking screenshots of outputs from pedigree creation programs, thus making the image uploading tool essential to their workflow. However, retrieval is more difficult due to images not being searchable. This suggests that it may be better to store these images in an image archival system and having a descriptive hyperlink to the image, instead of embedding them within the notes. Classification of images uploaded to the EHR allowed us to identify systems that are candidates for fuller integration with the EHR.

When examining which specialties uploaded clinical images, we found that the dermatology department used the image uploading tool the most in our sample. This was not surprising, considering that dermatology involves disorders that generally require understanding of visual changes [5]. However, it is clear that non-radiological images are relevant to multiple other specialties. For example, ophthalmology and surgery both contributed a significant number of images to our study. Additionally, 30 types of providers uploaded images. This finding indicates that image tools should be optimized for a variety of specialties and be accessible by people with different roles within the clinical workflow.

Given our results, we have the following recommendations for institutions using an EHR system that supports image upload:

1. There should be review of what is being uploaded to the system. During our study, we found many screenshots of the same systems within a specialty. If there was regular review of what is being uploaded, then knowledge is gained about how systems can be configured to support users' needs and what systems should be integrated in the EHR systems, in addition to any interoperability issues that there might be.

2. Users should be trained on when to consider adding photos to the record and how to take good clinical photos. In our review of notes, we found many patient notes that contained multiple images of the exact same site but with some photos out of focus ( Figure 3). To minimize noise and ensure that the relevant, most important image is being uploaded and viewed, users should understand what images are important and valuable.

3. Healthcare organizations should carefully consider how they distribute training and resources amongst those who utilize the image system highly and those who do not. In our study, we saw that three specialties uploaded over half of the clinical images that we examined ( $>$ Table 2). While basic training and functionality should be available to all, it was clear that certain specialties will utilize resources more than others.

4. Health systems should consider policies for uploading and protecting images. A particularly important question is whether providers can take photos using a personal smartphone and, if so, how they need to be protected on the phone and during transfer. Our hospital recently developed CliniCam, an iOS mobile application for taking clinical photographs and securely uploading those images onto patients' medical records,. During a preliminary usability study with resident physicians from emergency medicine, ambulatory internal medicine, and dermatology, they saw that $89 \%$ of users found the application useful or very useful for clinical practice [10]. The use of an automatic image-uploading tool such as CliniCam could affect what images get included or how those images are incorporated in EHRs. 


\section{Conclusion}

Analyzing images uploaded to an EHR system allowed us to characterize who uses the image uploading feature and what images are uploaded. Most uploaded images were non-clinical. Of the clinical images, photos and diagrams were the most common, physicians were the most common role type using this feature, and dermatologic images were the most common specialty. Screenshots of other systems illustrate the lack of interoperability between clinical information systems, but also highlight how systems can be improved. Understanding what is uploaded to an EHR system highlights ways to optimize provider usability to enhance documentation, workflow efficiency, diagnostic speed, and accuracy. Future work would include conducting demographic analysis on the providers who upload images and interviewing providers on the usefulness of such a system.

\section{Clinical Relevance Statement}

Images supplement and sometimes replace textual information in electronic patient health records. While examining the images uploaded to a home grown EHR, we found a great diversity in clinical images and their subject, including screenshots of other systems incompatible with the EHR. When institutions implement EHR systems, they must take into account the interoperability of that EHR with their other systems and whether or not the EHR has flexible image uploading and display to maximize utilization of clinical and non-clinical images. This study reinforces the importance of understanding how users utilize image uploading systems in a clinical setting

\section{Limitations}

Our study has several limitations. Our results represent data from outpatient care practices affiliated with a single large academic healthcare center using a locally-developed EHR system. Our data is from 2009-2010, so it would be interesting to examine how rapid changes in technology affect how providers interact with the images in their EHR. Additionally, we analyzed data from a single, homegrown EHR system, which could affect how generalizable our results are.

\section{Multiple Choice Question}

Which of the following statements is false?

A) Images provide important information in patient notes.

B) Many different provider types place images in notes.

C) Sites should not monitor what images are placed in notes.

D) People use images for different purposes.

The answer is C. Sites should monitor what images are placed in notes as that yields valuable information on who uses image uploading tools and on potential issues with their EHR system. In our study, we were able to understand who uses the image system the most, which allows us to identify potential candidates for additional resources. Additionally, we were able to find programs that were not integrated well into our EHR system by examining the images. By identifying these programs, we are able to better provide support for those who use these programs and work to integrate them within our EHR.

\section{Conflict of Interest}

The authors have no other competing interests or funding sources to disclose.

\section{Human Subjects Protection}

The study was performed in compliance with the World Medical Association Declaration of Helsinki on Ethical Principles for Medical Research Involving Human Subjects, and was reviewed and approved by the PHS Institutional Review Board. 


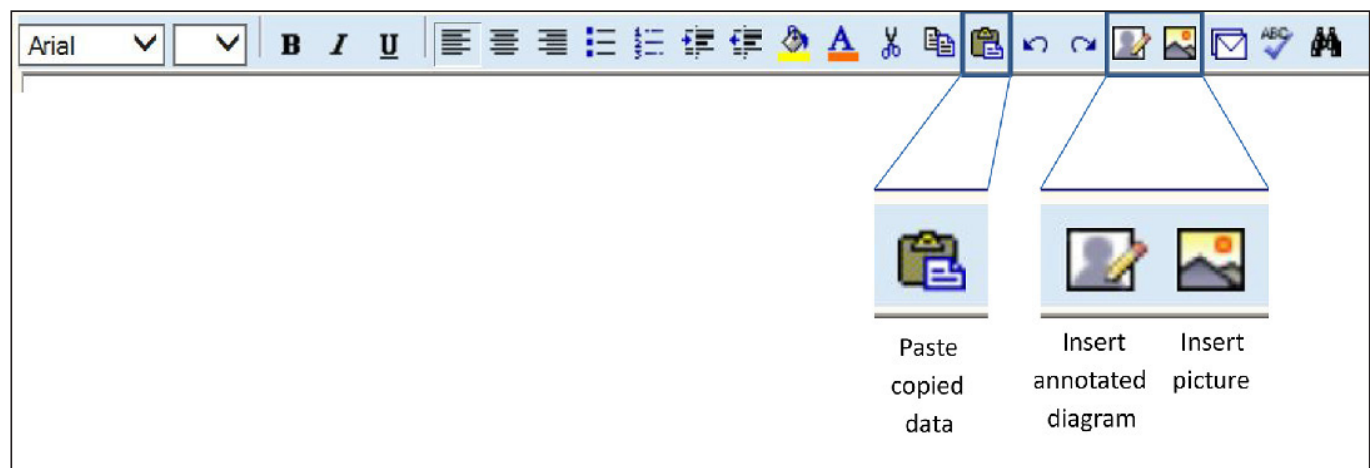

Fig. 1 Screenshot of toolbar within the note entry screen in the LMR. Arrows indicate ways of entering an image into the note.

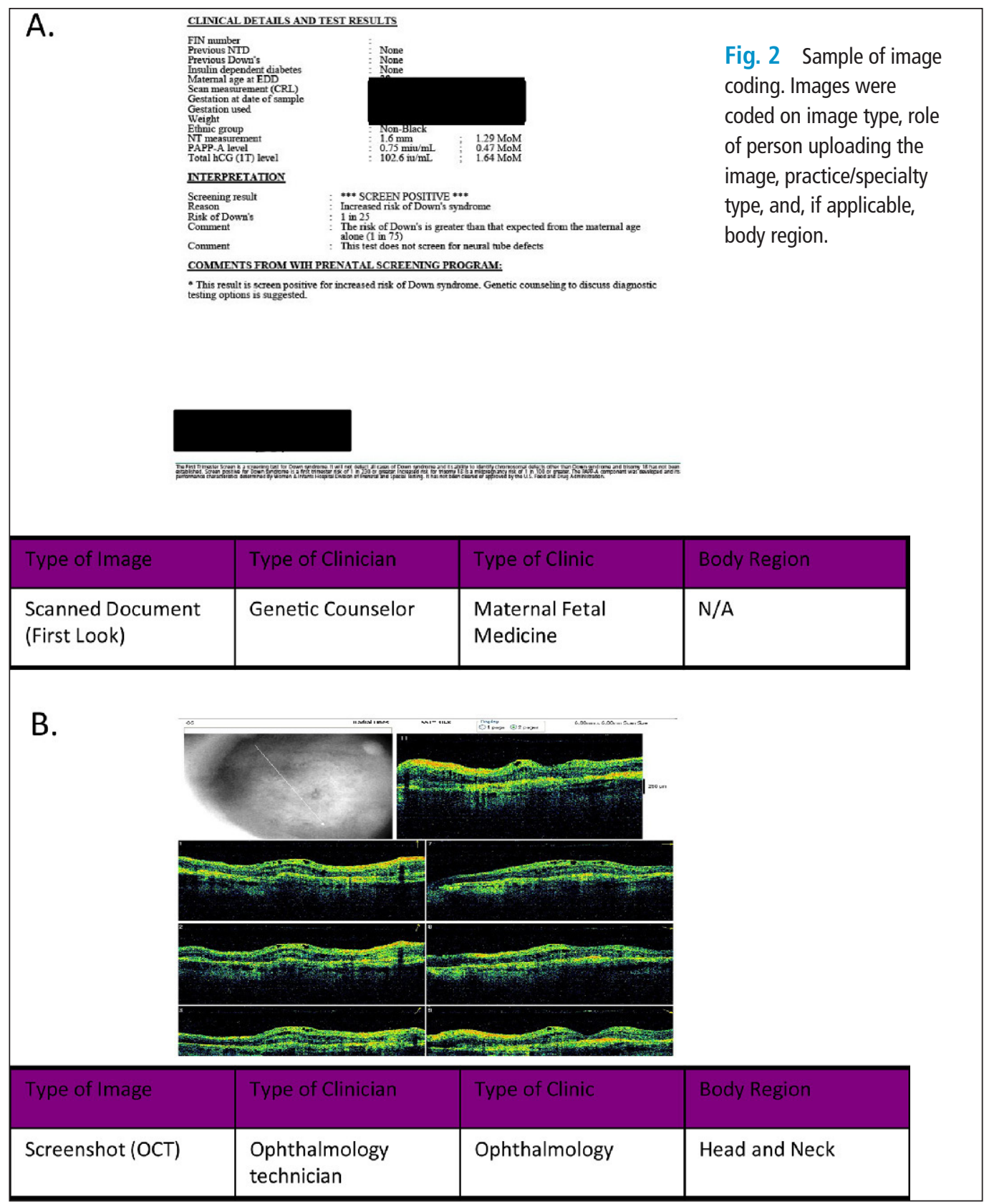




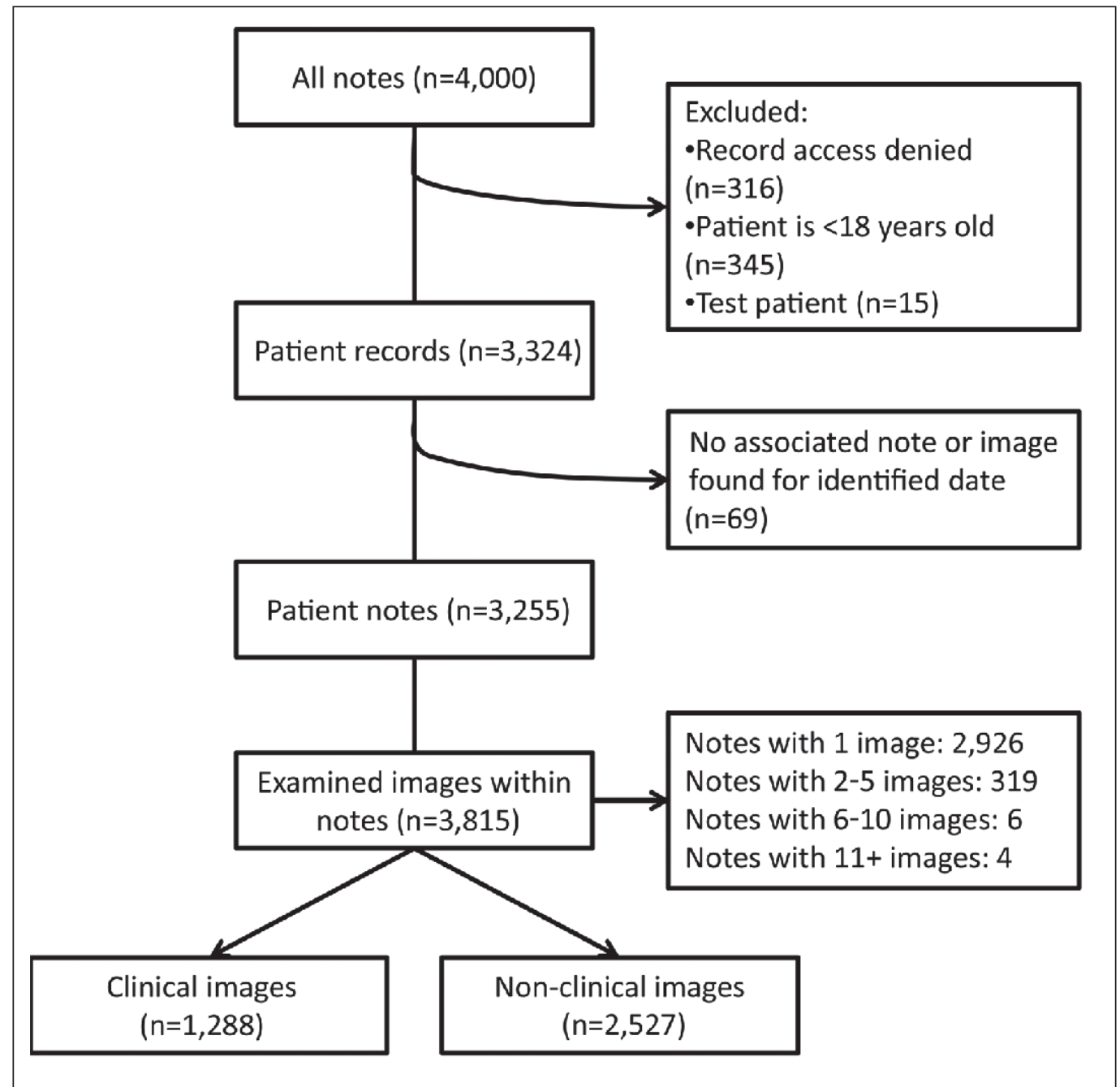

Fig. 3 Flow diagram of the notes and images analyzed. 


\begin{tabular}{|l|l|}
\hline Type of Images & $\mathbf{n}(\%$ of 3815$)$ \\
\hline Non-Clinical Images & $\mathbf{2 , 5 2 7}(\mathbf{6 6 . 2 \%})$ \\
\hline Logos & $2,304(91.2)$ \\
\hline Signatures & $212(8.4)$ \\
\hline Icon & $11(0.4)$ \\
\hline Clinical Images & $1,288(37.7 \%)$ \\
\hline Photos & $489(38.0)$ \\
\hline Diagrams & $246(19.1)$ \\
\hline Scanned Documents & $186(14.4)$ \\
\hline Graph & $141(11.0)$ \\
\hline Screenshots & $126(9.8)$ \\
\hline Table & $69(5.3)$ \\
\hline Notations & $24(1.9)$ \\
\hline Pedigrees & $5(0.4)$ \\
\hline Form & $2(0.2)$ \\
\hline
\end{tabular}

Table 1 Analysis of types of images uploaded to the LMR between July 1, 2009 and June 30, 2010. $\mathrm{N}=3815$.

\begin{tabular}{|l|l|}
\hline Type of Specialty & Clinical Images Uploaded $\mathbf{n}, \mathbf{( \% )}$ \\
\hline Dermatology & $450(34.9 \%)$ \\
\hline Ophthalmology & $208(16.1 \%)$ \\
\hline General Surgery & $139(10.8 \%)$ \\
\hline Obstetrics and Gynecology & $70(5.4 \%)$ \\
\hline Oncology & $65(5.0 \%)$ \\
\hline Maternal Fetal Medicine & $36(2.7 \%)$ \\
\hline Optometry & $35(2.7 \%)$ \\
\hline Internal Medicine & $33(2.6 \%)$ \\
\hline Cardiology & $25(1.9 \%)$ \\
\hline Genetic Counseling & $18(1.4 \%)$ \\
\hline Other & $209(16.2 \%)$ \\
\hline
\end{tabular}

Table 2 Top ten specialties uploading clinical images, determined by volume of clinical images. $\mathrm{N}=1,288$. 


\section{References}

1. Seto B, Friedman C. Moving toward multimedia electronic health records: how do we get there? J Am Med Inform Assoc 2012, 19(4): 503-505.

2. Ratib O, Swiernik M, McCoy JM. From PACS to integrated EMR. Comput Med Imaging Graph 2003, 27(2-3): 207-215.

3. Wiedemann LA: Using Clinical Photos in EHRs. J AHIMA 2010, 81(4): 44-45.

4. Lyon CC, Harrison PV. Digital imaging and teledermatology: educational and diagnostic applications of a portable digital imaging system for the trainee dermatologist. Clin Exp Dermatol 1997, 22(4): 163-165.

5. Windsor JS, Rodway GW, Middleton PM, McCarthy S. Digital photography. Postgrad Med J 2006, 82(972):688-692.

6. Miller MW, Ross RK, Voight C, Brouwer H, Karavite DJ, Gerber JS, Grundmeier RW, Coffin SE: Patientgenerated Digital Images after Pediatric Ambulatory Surgery. Appl Clin Inform 2016, 7(3): 646-652.

7. Dayhoff RE, Kuzmak PM, Kirin G, Frank S: Providing a complete online multimedia patient record. Proc AMIA Symp 1999: 241-245.

8. Albright D. Digital Leather Binding: The Rise and Fall of Skeumorphic Design [http://www.makeuseof. com/tag/digital-leather-binding-rise-fall-skeuomorphic-design/] 2015 (accessed January 20, 2016).

9. Campbell-Dollaghan K. Skeumorphism Will Never Go Away, and That's a Good Thing [http://gizmodo.com/skeuomorphism-will-never-go-away-and-thats-a-good-thin-1642089313] 2014 (accessed January 20, 2016).

10.Landman A, Emani S, Carlile N, Rosenthal DI, Semakov S, Pallin DJ, Poon EG: A mobile app for securely capturing and transferring clinical images to the electronic health record: description and preliminary usability study. JMIR Mhealth Uhealth 2015, 3(1): e1. 